\title{
The Treaty: Debating and Establishing the Irish State, Liam Weeks, Mícheál ó Fathartaigh (dir.)
}

\section{Philippe Cauvet}

\section{(2) OpenEdition}

1 Journals

\section{Édition électronique}

URL : https://journals.openedition.org/etudesirlandaises/8667

DOI : 10.4000/etudesirlandaises.8667

ISSN : 2259-8863

\section{Éditeur}

Presses universitaires de Caen

\section{Édition imprimée}

Date de publication : 31 décembre 2019

Pagination : 155-156

ISBN : 978-2-84133-964-8

ISSN : 0183-973X

\section{Référence électronique}

Philippe Cauvet, «The Treaty: Debating and Establishing the Irish State, Liam Weeks, Mícheál

Ó Fathartaigh (dir.) », Études irlandaises [En ligne], 44-2 | 2019, mis en ligne le 06 mai 2020, consulté le 15 novembre 2022. URL : http://journals.openedition.org/etudesirlandaises/8667 ; DOI : https:// doi.org/10.4000/etudesirlandaises.8667

\section{(c) $)(7)(2)$}

Creative Commons - Attribution - Pas d'Utilisation Commerciale - Partage dans les Mêmes Conditions 4.0 International - CC BY-NC-SA 4.0

https://creativecommons.org/licenses/by-nc-sa/4.0/ 


\title{
COMPTES RENDUS
}

\author{
BOOK REVIEWS
}

\author{
The Treaty: Debating and Establishing the Irish State, Liam Weeks, Mícheál \\ Ó Fathartaigh (dir.), Newbridge, Irish Academic Press, 2018, 324 p.
}

L'objectif de cet ouvrage collectif publié sous la direction de Liam Weeks et de Mícheál Ó Fathartaigh est de compléter la connaissance historique sur le Traité de 1921. En effet les deux directeurs de publication soulignent en introduction la relative absence de travaux historiques sur ce moment et sur ce document pourtant fondateurs de l'État irlandais. Seuls deux ouvrages, celui de Frank Pakenham, Peace by Ordeal, paru en 1935 et celui de Jason Knirck, Imagining Ireland's Independence, paru en 2006, couvraient cette période sans pour autant rentrer réellement dans l'analyse. À quelques mois du centenaire du Traité qui sera célébré en 2021, cette nouvelle publication arrive à point nommé pour combler un vide historiographique criant.

Le livre est composé de neuf chapitres distincts, chacun portant sur une thématique ou une problématique particulière; les contributions rassemblées ici apportent donc un regard nouveau sur le contexte politique, social et culturel dans lequel ce Traité a été débattu et ratifié par le deuxième Dáil, entre 1921 et 1922. Conformément à l'objectif initial annoncé en introduction par les directeurs de publication, ces contributions abordent des questions qui jusque-là ont souvent été négligées. Deux contributions (chapitres 6 et 7) creusent ainsi la question du rôle des femmes et de leur place dans les clivages politiques de l'époque. Deux autres soulèvent la question de la sociologie du mouvement républicain et des membres du Dáil (chapitres 4 et 5). Le chapitre 5 coécrit par Eunan O’Halpin et Mary Staines est particulièrement intéressant, puisqu'il s'agit de la biographie croisée du grand-père de l'une et de l'arrière-grand-père de l'autre, situés à l'époque de part et d'autre de l'échiquier politique! Un autre chapitre, lui aussi novateur, propose une analyse linguistique quantitative des débats au Dáil (chapitre 9).

De ces travaux, fort utiles, il ressort plusieurs leçons. La première, la plus importante, concerne les raisons du clivage entre les pro et les anti-Traité. Ce clivage ne correspondait ni à des clivages latents qui auraient préexisté au sein du républicanisme irlandais, ni à des appartenances de classes ou de genres. Rien ne permettait de prévoir l'orientation que prendraient ces débats. C'est peut-être pourquoi - et c'est une autre leçon du livre - l'interruption des débats pour la pause de Noël a joué un rôle considérable dans l'évolution des débats au Dáil. Ce livre démontre très clairement qu'au retour de cette pause, une tendance plus nette était apparue en faveur de l'acceptation du Traité, les membres du Dáil étant influencés assez sensiblement par leur électorat local, majoritairement pro-Traité. Ce sont donc les Irlandais autant que les membres du Dail qui ont choisi de ratifier 
le Traité obtenu quelques semaines plus tôt par Michael Collins et Arthur Griffith. En revanche, ce livre est peut-être moins convaincant quant à la leçon qu'il tire sur la question de la partition. D'ailleurs, l'on peut déplorer qu'aucune contribution ne soit consacrée à cette question, car, même si elle est restée mineure, les débats entre Seán Milroy et Seán MacEntee sur ce point auraient mérité d'être analysés et contextualisés. Tout en constatant que la question de la partition n'a pas du tout été centrale dans le clivage entre pro et anti-Traité (les débats ont principalement porté sur le statut du futur État irlandais et sur le serment d'allégeance à la Couronne), la conclusion se contente d'expliquer ce paradoxe en rappelant que le clivage entre unionisme ulstérien et nationalisme existait depuis déjà assez longtemps et que, implicitement au moins, l'idée partitionniste était acceptée, y compris dans les milieux nationalistes et au Sinn Féin. Or c'est précisément là que la question devient intéressante: d'où vient ce partitionnisme nationaliste et républicain? Comment expliquer que, tout en reconnaissant que la création de l'Irlande du Nord marque l'échec du nationalisme républicain irlandais, celui-ci ait pu finalement négliger cette question et, d'une certaine manière, laisser la partition se produire?

En somme, même s'il n'apporte pas toutes les réponses aux nombreuses questions historiques que soulève la ratification du Traité de 1921, la lecture de cet ouvrage, riche et dense, est vivement conseillée à tout historien et à tout étudiant souhaitant analyser en profondeur l'histoire du républicanisme et la fondation de l'État irlandais.

Philippe Cauvet

\section{Janet Mullarney, Catherine Marshall, Mary Ryder (eds.), Newbridge, Irish Academic Press, 2019, 222 p.}

Catherine Marshall and Mary Ryder's book on Janet Mullarney (b. 1952, Dublin) aims at archiving the artist's work over more than fifty years, from 1962 to 2018. It features essays by famous art historians, brief texts from friends of the artist as well as architects she has worked with, a complete catalogue of her works (p. 162-213) and a biography/ select bibliography (p. 214-220). The numerous colour plates allow the readers to get a precise idea of the evolution of the artist's work and bear witness to her preoccupation with materiality, light and space. Janet Mullarney was born in Ireland, travelled across Europe before settling in Tuscany, where her house and studio are peopled with her hybrid creatures. Rather than giving the readers a linear presentation of Janet Mullarney's life, works (sculptures, installations, drawings), or sources of inspiration, the authors share their impressions of her art, drawing a multi-faceted portrait of the artist.

In her essay, "All the Stances of Shyness" (p. 10-12), Catherine Marshall focuses on Mullarney's double geographical anchorage (in Ireland and Italy) before stressing the originality of her art: opting for figuration, wood carving and object-based sculpture meant going against the grain back in the 1980-1990s. Her art is neither 